\title{
Q2 Novel LC-MS/MS method for plasma vancomycin: Comparison with immunoassays and clinical impact
}

\author{
Q3 Matthijs Oyaert ${ }^{\text {a,* }}$, Nele Peersman ${ }^{a}$, Davy Kieffer ${ }^{\text {a,b }}$, Kathleen Deiteren ${ }^{\text {c }}$, Anne Smits ${ }^{\text {d,e }}$, Karel Allegaert ${ }^{\text {d,e }}$, \\ Isabel Spriet $^{\mathrm{f}, \mathrm{g}}$, Johan Van Eldere ${ }^{\mathrm{a}, \mathrm{b}}$, Jan Verhaegen ${ }^{\mathrm{a}, \mathrm{b}}$, Pieter Vermeersch ${ }^{\mathrm{a}, \mathrm{h}}$, Steven Pauwels ${ }^{\mathrm{a}, \mathrm{h}}$ \\ a University Hospitals Leuven, Department of Laboratory Medicine, B-3000 Leuven, Belgium \\ ${ }^{\mathrm{b}}$ KU Leuven - University of Leuven, Department of Microbiology and Immunology, B-3000 Leuven, Belgium \\ c Antwerp University Hospital, Department of Laboratory Medicine, Edegem, Belgium \\ ${ }^{\mathrm{d}}$ KU Leuven - University of Leuven, Department of Development and Regeneration, B-3000 Leuven, Belgium \\ e University Hospitals Leuven, Neonatal Intensive Care Unit, B-3000 Leuven, Belgium \\ ${ }^{\mathrm{f}}$ KU Leuven - University of Leuven, Department of Clinical Pharmacology and Pharmacotherapy, B-3000 Leuven, Belgium \\ g University Hospitals Leuven, Pharmacy Department, B-3000 Leuven, Belgium \\ ${ }^{\mathrm{h}}$ KU Leuven - University of Leuven, Department of Cardiovascular Sciences, B-3000 Leuven, Belgium
}

\section{A R T I C L E I N F O}

\section{Article history:}

Received 14 August 2014

Received in revised form 8 December 2014

Accepted 10 December 2014

Available online $\mathrm{xxxx}$

\section{Keywords:}

LC-MS/MS

Vancomycin

Tandem mass spectrometry

Therapeutic drug monitoring

\begin{abstract}
A B S T R A C T
Background: Accurate quantification of vancomycin in plasma is important for adequate dose-adjustment. 24 As literature suggests between-method differences, our first objective was to develop a novel liquid 25 chromatography-tandem mass spectrometry (LC-MS/MS) method for total vancomycin in human plasma and to 26 compare frequently used immunoassays with this method. Secondly, we investigated the clinical impact of 27 between-method quantification differences.

Methods: For LC-MS/MS, lithium heparin plasma was extracted by adding a precipitation reagent containing the in- 29 ternal standard (vancomycin-des-leucine). Analysis was performed on an Acquity TQD mass spectrometer 30 equipped with an Acquity UPLC 2795 separations module. Our method was analytically validated and compared 31 with four frequently used immunoassays from four different manufacturers. Vancomycin concentrations were 32 clinically classified as toxic, therapeutic and sub-therapeutic. Clinical discordance was calculated using LC-MS/MS 33 as a reference.

Results: A novel LC-MS/MS method using protein precipitation as sole pretreatment and an analysis time of 5.0 min 35 was developed. The assay had a total imprecision of $2.6-8.5 \%$, a limit of quantification of $0.3 \mathrm{mg} / \mathrm{L}$ and an accuracy 36 ranging from 101.4 to $111.2 \%$. Using LC-MS/MS as reference, three immunoassays showed a mean proportional 37 difference within $10 \%$ and one showed a substantial mean proportional difference of $>20 \%$. Clinical discordant 38 interpretation of the obtained concentrations ranged from 6.1 to $22.2 \%$.

Conclusions: We developed a novel LC-MS/MS method for rapid analysis of total vancomycin concentrations in 40 human plasma. Correlation of the method with immunoassays showed a mean proportional difference $>20 \%$ for 41 one of the assays, causing discordant clinical interpretation in more than 1 out of 5 samples.
\end{abstract}

(c) 2014 Published by Elsevier B.V. 43

\section{Introduction}

Vancomycin is a glycopeptide antibiotic with strong bactericidal activity against gram-positive bacteria. These do not only include methicillin-resistant Staphylococci, but also penicillin resistant organisms, such as Streptococci and Corynebacteria [1]. Large inter- and

Abbreviations: LC-MS/MS, liquid chromatography-tandem mass spectrometry; TDM, therapeutic drug monitoring; IS, internal standard; QC, quality control; MRM, multiple reaction monitoring; $\mathrm{S} / \mathrm{N}$, signal to noise ratio; $\mathrm{CV}$, coefficient of variation; $\mathrm{ME}$, matrix effect; $\mathrm{DR}$, detection range; FPIA, fluoro polarized immuno assay; PETINIA, particle enhanced turbidimetric inhibition immunoassay; LOQ limit of quantification; LOA, limit of agreement

* Corresponding author at: University Hospitals Leuven, Laboratory Medicine, Herestraat 49, B-3000 Leuven, Belgium. Tel.: + 32163470 00; fax: + 3216347931 .

E-mail address: matthijsoyaert@telenet.be (M. Oyaert). intra-patient variability, combined with a correlation between low 53 plasma concentrations and therapeutic failure on the one hand, and 54 high plasma concentrations and toxicity on the other hand, makes the 55 molecule an excellent candidate for therapeutic drug monitoring 56 (TDM). In addition, the potential rise in minimum inhibitory concentra- 57 tions of vancomycin target organisms makes it increasingly important 58 to adjust its dosage in order to ensure adequate concentrations [2,3]. 59 In clinical practice, therapeutic intervals, target levels and dose- 60 adjustment schemes in function of administration mode and sampling 61 time are used.

Current recommendations, however, do not take into account that 63 routine plasma vancomycin quantification by commercial immunoas- 64 says can show substantial between-method differences [4-6]. Next to 65 standardization issues, immunoassays can also lack specificity. For 66 
example, cross-reacting substances such as vancomycin degradation products have been described to interfere with some immunoassays [7]. Also, several structurally related compounds are formed during the production process and can be present in the isolated substance. A study of Diana et al. investigated the impurities present in a commercial vancomycin sample and found 15 different impurities, together composing $16.6 \%$ of the sample [7]. The clinical impact of these issues was recently suggested in a paper by Zhao et al., in which the predictive performances of different neonatal pharmacokinetic models for vancomycin administration were compared [8]. They found different predictive performances between different analytical methods for serum vancomycin concentrations, thereby highlighting that dosage individualization of vancomycin in neonates should not only consider patients' characteristics like body weight, but also the methods used to measure vancomycin [9]. Moreover, it remains often difficult to track the analytical details of the methods used to measure vancomycin in determining therapeutic intervals and target values [10], thereby shedding doubt on the applicability of the guidelines in specific hospital settings. Lastly, current guidelines use the total concentration of vancomycin (free and bound) for dosage adjustment [11], even though it is known that, as for most antibiotics [12], it is probably the free concentration that is critical for diffusion into infected areas [13,14]. Whether reported proteinbinding percentages for vancomycin are stable and predictable when only looking at total vancomycin concentrations is part of an ongoing discussion. The same holds true for the added value of measuring free concentrations.

To tackle the above-mentioned limitations, a number of methods using mass spectrometry (LC-MS/MS) for the quantification of plasma total vancomycin concentration have been described. These methods, however, rely on internal standard (IS) compounds that are structurally not related to the target analyte (teicoplanin, atenolol, kanamycin-B) [15-18], use a labor-intensive sample preparation [5,16], or have very long runtimes [5].

It is known that the IS has a crucial role in compensating for sample specific matrix effects (MEs) in LC-MS/MS assays. As most studies relied on other, structurally and hence physically and chemically unrelated compounds as IS, it is not surprising that significantly different percentages of ME between vancomycin and IS (up to $50 \%$ difference [18]) have been described in published methods, shedding serious doubt on the quantification accuracy of clinical samples presenting with varying matrices. A recently published method tried to cope with this problem by synthesizing a homemade vancomycin derivative [5]. Although this method was intended as a reference method, the use of a homemade vancomycin derivative as IS is time-consuming and offers no workable solution for other groups trying to easily measure vancomyin with mass spectrometry. Moreover, this method has an analysis time of up to 20 min per sample [5].

The first aim of our study was to develop a novel LC-MS/MS method for measuring total vancomycin concentrations with acceptable runtimes and using an adequate IS. To chart between-method differences, we compared four frequently used immunoassays with our method. In addition, we investigated the clinical impact of the observed differences.

\section{Materials and methods}

\subsection{Chemicals and solutions}

Vancomycin $\mathrm{HCl}$ was purchased from Toronto Research Chemicals (Toronto, Canada). Vancomycin-des-leucine formiate was purchased from Alsachim (Strasbourg, France; formulation on request) and acetonitrile (LC-MS grade) from BioSolve (Valkenswaard, The Netherlands). HPLC-grade water was generated using a Milli-Q-water-purification system (Millipore, Molsheim, France). Pooled blank lithium heparin (Becton Dickinson, Franklin Lakes, USA) blood samples were collected from a healthy volunteer.
A stock solution of vancomyin in water at $4.0 \mathrm{mg} / \mathrm{mL}$ was prepared. 130 Ten calibration standards at vancomycin concentrations of 0.6, 1.3, 2.5, 131 5.0, 10.0, 20.0, 30.0, 50.0, 75.0 and $100.0 \mathrm{mg} / \mathrm{L}$ were prepared by appro- 132 priate addition of stock solution to the blank plasma pool. An IS working 133 solution of $5.0 \mathrm{mg} / \mathrm{L}$ vancomycin des-leucine in Milli-Q was used. In 134 each routine analysis, four levels of quality control (QC) (3.0, 15.0, 135 30.0 and $75.0 \mathrm{mg} / \mathrm{L}$ ) were analyzed. These were prepared by the appro- 136 priate addition of another (independently prepared and weighed) stock 137 of vancomycin $(4.0 \mathrm{mg} / \mathrm{mL}$ in water) to blank pooled plasma. QCs, 138 calibration standards and IS working solution were stored at $-20{ }^{\circ} \mathrm{C} 139$ until use.

\subsection{Sample preparation and LC-MS/MS conditions}

141

Lithium heparin blood samples were centrifuged for 10 min at $1912 \mathrm{~g}$. 142 $40 \mu \mathrm{L}$ plasma was immediately vortexed with $40 \mu \mathrm{L}$ IS working solution 143 and $160 \mu \mathrm{L}$ acetonitrile in glass tubes. After centrifugation (10 min at 144 $16,100 \mathrm{~g}$ ), $5 \mu \mathrm{L}$ of supernatant was injected (auto-sampler) into the chro- 145 matographic system. Chromatographic separation was carried out on an 146 Acquity UPLC separations module (Waters Ltd, Watford, UK). As analyti- 147 cal column, an Acquity UPLC BEH HILIC ( $100 \mathrm{~mm} \times 2.1 \mathrm{~mm}$; $1.7 \mu \mathrm{m}, 148$ Waters Ltd, Watford, UK), maintained at $50{ }^{\circ} \mathrm{C}$, was used with a 149 Phenomenex C-18 guard column (100 mm $\times 4 \mathrm{~mm}$, Torrance, CA, USA) 150 as pre-column.

The mobile phase was a mixture of acetonitrile (buffer A) and water 152 (buffer B) both containing 0.1\% formic acid. A linear gradient starting 153 from $95 \%$ buffer A descending to $40 \%$ buffer A at 2.50 min was applied. 154 At $2.60 \mathrm{~min}$, buffer A was set at 99\% and kept till $4.00 \mathrm{~min}$. From 4.00155 to $5.00 \mathrm{~min} 95 \%$ buffer A was used to re-equilibrate for the next injec- 156 tion. The flow rate was set at $0.45 \mathrm{~mL} / \mathrm{min}$, the total runtime was 157 $5.0 \mathrm{~min}$. Mass spectrometric analysis was performed using a tandem 158 mass spectrometer (Acquity TQD detector, Waters Ltd, Watford, UK) 159 equipped with an electrospray ionization source operating in the 160 electrospray-positive mode. The source and desolvation temperature 161 were set at $150{ }^{\circ} \mathrm{C}$ and $500{ }^{\circ} \mathrm{C}$, respectively. Nitrogen was used as 162 desolvation gas and was set at a flow rate of $750 \mathrm{~L} / \mathrm{h}$. Capillary voltage 163 was set at $3.5 \mathrm{kV}$, cone voltage at $20 \mathrm{~V}$ and collision energy at $20 \mathrm{eV}$. Van- 164 comycin was detected by multiple reaction monitoring (MRM) with a 165 dwell time of $0.085 \mathrm{~s}$. The following MRM transitions were monitored: 166 $\mathrm{m} / \mathrm{z} 725.2 \rightarrow 144.0$ and $726.1 \rightarrow 144.0$ for vancomycin, and 167 $662.1 \rightarrow 144.0$ for vancomycin des-leucine. Vancomycin was quantified 168 by means of calibration to each run, using a weighted least square $\left(1 / X^{2}\right) 169$ regression in MassLynx software (Waters Ltd, Watford, UK) of the 10170 calibration standards. For vancomycin the 2 MRMs were summed. 171

\subsection{Analytical validation}

Method imprecision was evaluated by analysis of four QC concentra- 173 tions and three concentrations of patient samples on ten consecutive 174 days [19]. A total imprecision of $<15 \%$ was acceptable [20]. 175

The limit of quantification (LOQ) was defined as the lowest analyte 176 concentration with a signal to noise ratio $(\mathrm{S} / \mathrm{N})$ of more than 10 , a 177 coefficient of variation (CV) and accuracy $\leq 20 \%$ [21].

Linearity was evaluated by comparing if higher order equations give 179 significantly better fits using Microsoft Excel Analyse-it software. To 180 determine the amount of carry-over, we analyzed in the sequence 181 HHHBBB, where $\mathrm{H}$ is the highest calibration standard and $\mathrm{B}$ is a 182 blank. The percentage of carry-over was calculated with the formula 183 $100 \times($ B1 - B3) / (H3 - B3) [19].

Accuracy was calculated from the QC samples $(n=4)$ in ten differ- 185 ent runs as the percentage deviation from the theoretically added 186 vancomycin concentration. An accuracy of $<15 \%$ was accepted [20]. To 187 the best of our knowledge, no reference plasma exists for vancomycin. 188

Freeze and thaw, short-term, and long-term stability of plasma sam- 189 ples were determined at three concentration levels. Freeze and thaw 190 stability was tested by comparing freshly prepared samples to samples 191 
that underwent six freeze-thaw cycles. Short and long-term stability was assessed by storing samples at $20^{\circ} \mathrm{C}, 8^{\circ} \mathrm{C}$ and $-20^{\circ} \mathrm{C}$. The obtained results were compared with the results found in the initial analytical run. For each quantification, a fresh calibration curve was used. Extract stability was determined by measuring three vancomycin concentrations $(5,20$ and $50 \mathrm{mg} / \mathrm{L}) 24$ and $48 \mathrm{~h}$ after preparation, stored in the autosampler $\left(2-8{ }^{\circ} \mathrm{C}\right)$. A deviation of $\pm 20 \%$ compared to the results obtained in the initial run was accepted.

Extraction recovery was evaluated by comparing the peak areas of vancomycin, spiked in blank plasma, before and after extraction for three concentration levels (5, 20 and $40 \mathrm{mg} / \mathrm{L}$ ). We evaluated ME by comparing the peak areas of vancomycin spiked at 5 and $20 \mathrm{mg} / \mathrm{L}$ in pure solvent (water), with the peak areas of vancomycin spiked at 5 and $20 \mathrm{mg} / \mathrm{L}$ in six different blank plasma extracts (three patients who received a multitude of medication, but not vancomycin; two samples of healthy volunteers and one plasma filtrate). The sample ME was calculated with the equation $\mathrm{ME} \%=\mathrm{B} / \mathrm{A} * 100$, where $\mathrm{B}$ refers to the peak area of vancomycin obtained in matrix and $A$ to the peak area in solvent.

Selectivity was tested by running four samples from critically ill and hemato-oncology patients not receiving vancomycin, but a number of other frequently used medications (in total 39 other medications). The resulting chromatograms were examined for interfering peaks at the retention time of vancomycin.

\subsection{Method comparison}

Leftovers from lithium-heparin patient blood samples sent to the Clinical Laboratory of University Hospitals Leuven for clinically indicated vancomycin plasma measurements were used for method comparison (99 samples including 68 different patients and one external QC sample). Our study was performed with full respect for individuals' rights to confidentiality and in accordance with procedures supervised by the local authorities responsible for ethical research. Five aliquots of these samples were prepared and stored at $-20^{\circ} \mathrm{C}$ until analysis.

Total vancomycin plasma concentrations were measured with our LC-MS/MS assay and with four different immunoassay reagent systems. The vancomycin assay on Architect i2000SR (Abbott, North Chicago, Illinois, USA; detection range (DR): 3.0 to $100.0 \mathrm{mg} / \mathrm{L}, \mathrm{n}=98$ samples analyzed) is an immunoassay based on chemiluminescence using acridinium-conjugated antibodies (Fluoro Polarized Immuno Assay (FPIA)). The vancomycin assay on the Roche Cobas 8000 c702 (Roche Diagnostics, Mannheim, Germany; DR: 1.7 to $80.0 \mathrm{mg} / \mathrm{L} ; \mathrm{n}=99$ samples analyzed) and Ortho Vitros 5000 (Ortho Clinical Diagnostics, Mongkok, Hong Kong; DR: 5.0 to 100.0 mg/L; $\mathrm{n}=99$ samples analyzed) are based on the competition between the drug in a sample and the drug labeled with the enzyme glucose-6-phosphate-dehydrogenase, which is included in the assay for antibody binding sites. The Siemens Dimension Vista 1500 assay (Siemens Healthcare Diagnostics, Deerfield, IL; DR: $0.8-50.0 \mathrm{mg} / \mathrm{L} ; \mathrm{n}=98$ samples analyzed) is a homogeneous particle enhanced turbidimetric inhibition immunoassay (PETINIA) that incorporates a monoclonal detection antibody. Intra- and inter-run coefficients of variation (CV) were evaluated by paired analysis of at least ten different runs. The repeatability ( 1.6 to $3.0 \%, 1.5$ to $3.3 \%, 2.7$ to $3.1 \%$; and 1.5 to $3.4 \%$ ) and total imprecision assay CVs (3.1 to $6.2 \%$; 2.4 to $4.4 \%$; 5.1 to $6.1 \%$; and 2.7 to $3.9 \%$ ) on respectively Abbott Architect i2000SR, Roche Cobas 8000, Ortho Vitros 5000 and Siemens Dimension Vista 1500 , performed on internal quality control material, were satisfactory according to the respective leaflets [23-26].

To study the clinical impact of the vancomycin results obtained with the five different assays, we used the therapeutic range for vancomycin administered by continuous infusion $(15.0-25.0 \mathrm{mg} / \mathrm{L})[10,11]$ and classified the results as therapeutic $(15.0-25.0 \mathrm{mg} / \mathrm{L})$, toxic $(>25.0 \mathrm{mg} / \mathrm{L})$ or sub-therapeutic $(<15.0 \mathrm{mg} / \mathrm{L})$.

\subsection{Statistics}

Bland-Altman analysis, Passing Bablok regression analysis and 256 Pearson's correlation coefficients were used to study interchangeability 257 between results from the different immunoassays with LC-MS/MS as a 258 reference $[27,28]$. Data-analysis was performed by using Microsoft 259 Excel Analyse-it version 2.21 (Analyse-it Software Ltd, Leeds, UK).

\section{Results}

\subsection{Analytical validation}

Under the applied chromatographic conditions, vancomycin eluted 263 as a peak at a retention time of $2.7 \mathrm{~min}$. Chromatograms of a low and 264 high QC and a patient sample $(14.3 \mathrm{mg} / \mathrm{L})$ are shown in Fig. 1. Repeat- 265 ability and total imprecision were found to be $<9 \%$, (Table 1 ). The LOQ 266 was $0.3 \mathrm{mg} / \mathrm{L}$; the $\mathrm{CV}$ at this concentration was only $8.8 \%$. The assay 267 was linear in from 0.3 to $100.0 \mathrm{mg} / \mathrm{L}$. No significant carry-over was 268 detected $(<0.5 \%)$.

The accuracy ranged from 101.4 to $111.2 \%$ for the four QC concentra- 270 tions and was therefore within preset limits ( $<15.0 \%)$. Samples showed 271 no substantial degradation for short (one week) and long term (three 272 weeks) stability at different temperatures (Table 2). Concentrations 273 obtained after six freeze thaw cycles resulted in residual vancomycin 274 concentrations ranging from 95.6 to $101.9 \%$ compared to the initial 275 vancomycin concentration determined on fresh plasma. Extract stability 276 recovery for the three tested concentrations ranged from 86.0 to $114.0 \% 277$ after 24 and $48 \mathrm{~h}$, indicating acceptable processed sample stability of 278 our method for at least $48 \mathrm{~h}$.

The average extraction recovery was $106.3 \%$ (4.8\% CV) for vancomy- 280 cin. The ME ranged from 9.1 to $118.2 \%$ (47.2\% CV) and 34.2 to $85.9 \% 281$ ( $34.7 \% \mathrm{CV}$ ) for the 5 and $20 \mathrm{mg} / \mathrm{L}$ spiked vancomycin concentrations, re- 282 spectively. When the response ratios (RR) (area vancomycin/area IS) 283 were calculated for the different matrices, these ranged from 86.4 to 284 $117.0 \%$ (9.4\% CV) and 83.6-113.9\% (10.9\% CV) (5 and $20 \mathrm{mg} / \mathrm{L}$ vancomy- 285 cin, respectively) as compared to the RR in pure solvent. The results for 286 the different matrices are presented more in detail in Table 3. Matrix 287 seven was the only matrix that showed ion enhancement. Compared 288 to the other matrices that were taken on lithium heparin, this blood 289 sample was taken on a serum separator tube with gel and clot activator, 290 routinely not used for TDM of vancomycin. If the serum separator ma- 291 trix is omitted from analysis, the CV on RR further improves to $<8.5 \% 292$ for both levels. Of the $12 \mathrm{RR}, 2$ showed a ME (and hence accuracy) mar- 293 ginally worse than $\pm 15 \%$ [20]. One was $-16.4 \%$ at $20 \mathrm{mg} / \mathrm{L}$ and was 294 taken on the serum separator tube, which is not routinely used; the 295 other was $+17.0 \%$ at $5 \mathrm{mg} / \mathrm{L}$ and was seen in a matrix of a patient that 296 showed very substantial ion suppression. In our experience, such a re- 297 markable suppression is encountered very rarely. During our method 298 comparison, such low areas with respect to the calculated concentra- 299 tions were not observed. The matrix was that of a patient receiving a 300 multitude of medications (except vancomycin). The ME on the 301 $20 \mathrm{mg} / \mathrm{L}$ experiment of this patient was 105\% and well within limits, 302 so a pipetting error cannot be excluded either. As small amounts of 303 stock solution are spiked to the blank plasmas, relatively small pipetting 304 errors can yield more substantial deviations in accuracy and 305 imprecision.

306

The analysis of four plasma samples from patients that were on 307 medication other than vancomycin proved the selectivity of the 308 method; no peak signals in the retention time windows of vancomycin 309 or vancomycin des-leucine were observed.

\subsection{Method comparison}

Bland-Altman, Passing \& Bablok regression analysis and Pearson's 312 correlation coefficients were used to compare the four immunoassays 313 to our LC-MS/MS assay (Fig. 2). The regression indicated a proportional 314 
Relative Intensity

A
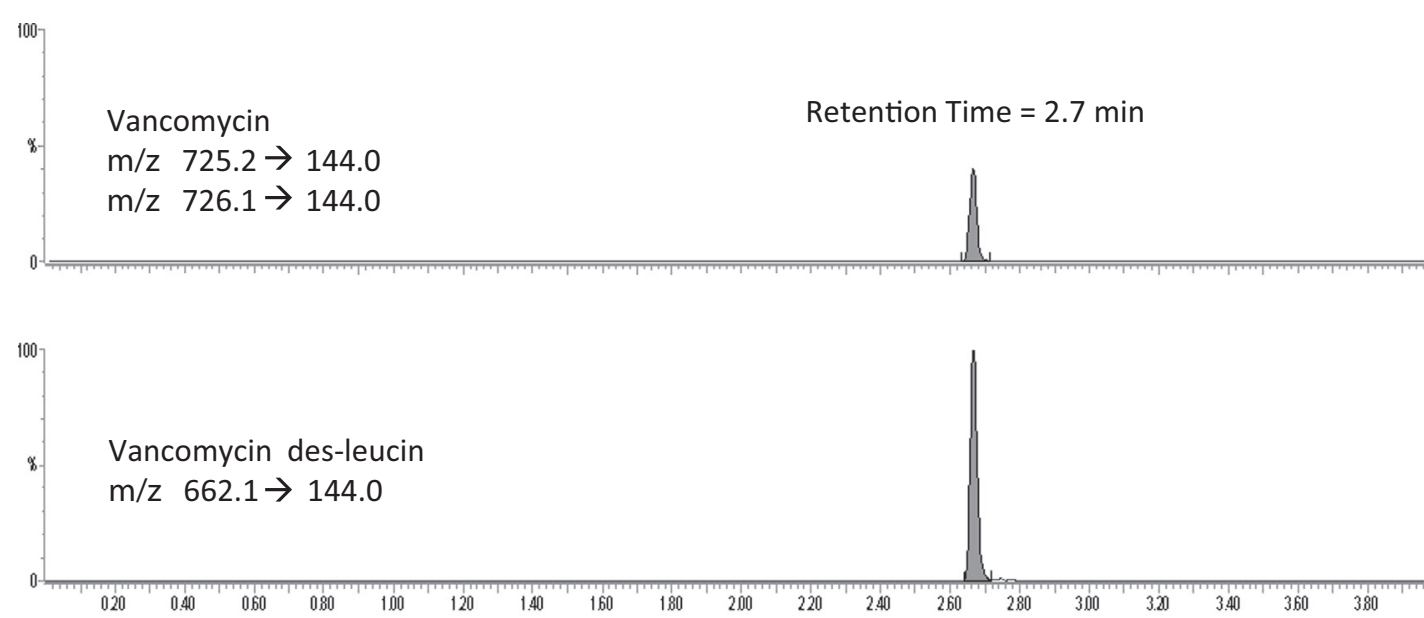

B
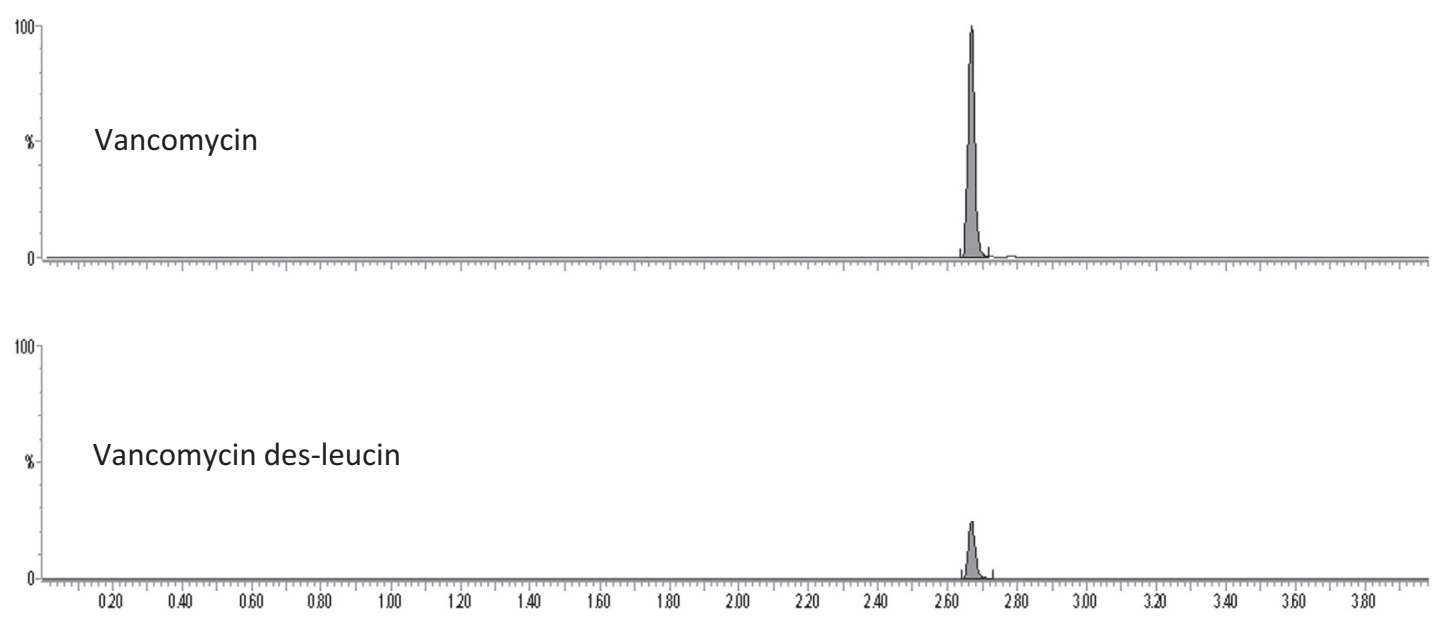

C
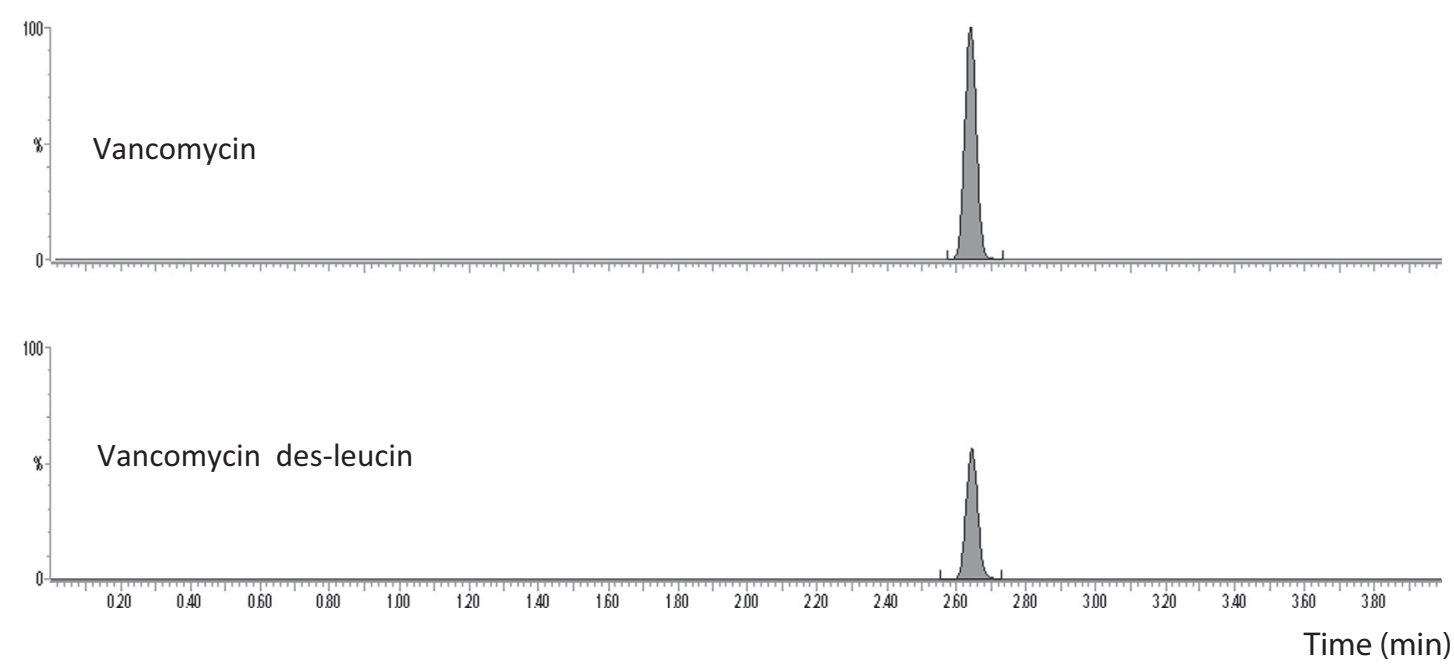

Fig. 1. A) Chromatogram of a low QC (3.0 mg/L vancomycin); B) chromatogram of a high QC (30.0 mg/L vancomycin); C) chromatogram of a patient sample (14.3 mg/L) vancomycin.

mean difference well within $10 \%$ for 3 out of 4 immunoassays. Only the Cobas 8000 assay showed a substantial proportional difference of $+22 \%$ as compared to LC-MS/MS. Using Bland-Altman analysis, the highest mean difference was found for the Roche Cobas assay (19.3\%; 95\%Cl: 16.5-22.0\%) (Fig. 2). Inter-immunoassay Bland-Altman mean differences, Passing \& Bablok regression analysis and Pearson's correla- 320 tion coefficients are presented in Supplemental Table 1.

In the Bland-Altman analysis, the 95\% limits of agreement (LOA) for 322 the inter-immunoassay comparisons show ranges spanning 31.9\%- 323 $48.4 \%$, whereas the $95 \%$ LOAs for comparison of immunoassays with 324 
t1.1 Table 1

t1.2 Imprecision of vancomycin LC-MS/MS analysis.

$\mathrm{t} 1.3$

$\mathrm{t} 1.4$

$\mathrm{t} 1.5$ distribution of the results is illustrated in Fig. 3.

\section{Discussion} behavior.

Table 2

\begin{tabular}{|c|c|c|c|c|c|c|}
\hline & \multicolumn{3}{|c|}{ Within-run imprecision } & \multicolumn{3}{|c|}{ Total imprecision } \\
\hline & Mean $(\mathrm{mg} / \mathrm{L})$ & $\mathrm{SD}(\mathrm{mg} / \mathrm{L})$ & $\% \mathrm{CV}$ & Mean (mg/L) & $\mathrm{SD}(\mathrm{mg} / \mathrm{L})$ & $\% \mathrm{CV}$ \\
\hline Low QC & 2.6 & 0.1 & 5.2 & 2.6 & 0.2 & 6.2 \\
\hline Medium QC & 14.0 & 0.5 & 3.7 & 12.7 & 1.1 & 8.5 \\
\hline High QC & 29.3 & 1.0 & 3.9 & 26.7 & 1.8 & 6.6 \\
\hline Ultra-high QC & 75.8 & 2.0 & 2.8 & 70.3 & 5.5 & 7.9 \\
\hline Patient low & 7.9 & 0.2 & 2.5 & 5.0 & 0.1 & 2.9 \\
\hline Patient medium & 19.5 & 0.6 & 2.9 & 14.0 & 0.7 & 5.2 \\
\hline Patient high & 48.9 & 1.7 & 3.5 & 27.8 & 0.7 & 2.6 \\
\hline
\end{tabular}

LC-MS/MS give ranges spanning $47.6 \%-57 \%$. This was not caused by a higher LC-MS/MS CV as the CVs on patient as well as QC samples (spiked plasma) for our method (2.6-8.5\% on plasma) are not substantially different from the CVs reported in the method insert for the immunoassays (2.4-6.2\% on iQC material). Residual ME in the LC-MS/MS method could be part of the explanation. The CV on the lithium heparin matrix RR, however, was $<8.5 \%$ and for immunoassays no such analysis has been reported, so it remains difficult to judge the relative impact. Small calibration differences might also add to the larger LOA span.

Not surprisingly, clinical interpretation of the obtained concentrations with the different assays showed that the largest deviation with respect to the LC-MS/MS assay was found for the Cobas 8000 immunoassay (22.2\% discordance). On the contrary, only $12.2 \%, 8.1 \%$ and $6.1 \%$ discordance was found between the Architect i2000SR, Vitros 5000 and Dimension Vista 15000 compared to LC-MS/MS, respectively. The

We developed and validated a novel LC-MS/MS method for quantification of total vancomycin in human plasma. All validation parameters were within the preset specifications. As our method was designed to be as user-friendly as possible, labor-intensive sample clean-ups and long runtimes to cope with matrix effects were avoided. The only sample pretreatment was a simple protein precipitation and the total analysis time was $5.0 \mathrm{~min}$. The use of a hydrophilic interaction column (HILIC) ensured adequate retention of vancomycin. We also managed to avoid a protein precipitation with trifluoro/trichloro-acetic acid as described by previous groups $[5,17,18]$. In our experience, the use of strong acids is not beneficial to the life span of the chromatographic column, the tubings and the chromatographic system in general. Not surprisingly, we observed substantial matrix effects. Our results show undesirable accuracy and large variation on the vancomycin areas in different matrices. An impressive improvement was seen when the RR was used (from mean accuracy about $67 \%$ with a CV about $40 \%$ to a mean accuracy about $102 \%$ with a CV about $11 \%$ ), indicating compensation by the IS. In contrast with other LC-MS/MS methods for vancomycin quantification [15-18], the structure of our IS was almost identical to that of the target analyte and ensured similar ionization and chromatographic

Commercially available automated immunoassays are widely used to quantify total vancomycin in serum or plasma. Although immunoassays

Table 3

Matrix effects (ME) and CVs for vancomycin LC-MS/MS analysis for 6 different matrices Q1 and water (matrix 1) for the A) $5 \mathrm{mg} / \mathrm{L}$ and B) $20 \mathrm{mg} / \mathrm{L}$ vancomycin experiments. ME for $\mathrm{t} 3.3$ vancomycin and IS are presented as a percentage of the area obtained in water. ME for $\mathrm{t} 3.4$ the response ratio (RR) was calculated as a percentage compared to the obtained RR in t3.5 water. The ME expressed as a percentage of pure solvent area or RR was used as accuracy. t3.6

\begin{tabular}{|c|c|c|c|c|c|c|c|}
\hline \multirow[t]{3}{*}{ A } & \multicolumn{6}{|c|}{$5 \mathrm{mg} / \mathrm{L}$ vancomycin experiment } & $\mathrm{t} 3.7$ \\
\hline & \multicolumn{2}{|c|}{ Vancomycin } & \multicolumn{2}{|l|}{ IS } & \multicolumn{2}{|l|}{ RR } & $\mathrm{t} 3.8$ \\
\hline & Area & ME & Area & $\mathrm{ME}$ & $\mathrm{RR}$ & ME & $\mathrm{t} 3.9$ \\
\hline Matrix 1 & 1257 & - & 1313 & - & 0.963 & - & $\mathrm{t} 3.10$ \\
\hline Matrix 2 & 898 & $71.5 \%$ & 860 & $65.5 \%$ & 1.047 & $108.8 \%$ & $\mathrm{t} 3.11$ \\
\hline Matrix 3 & 926 & $73.7 \%$ & 901 & $68.6 \%$ & 1.023 & $106.3 \%$ & $\mathrm{t} 3.12$ \\
\hline Matrix 4 & 1149 & $91.4 \%$ & 1231 & $93.8 \%$ & 0.934 & $97.0 \%$ & $\mathrm{t} 3.13$ \\
\hline Matrix 5 & 724 & $57.6 \%$ & 707 & $53.9 \%$ & 1.024 & $106.4 \%$ & $\mathrm{t} 3.14$ \\
\hline Matrix 6 & 115 & $9.1 \%$ & 102 & $7.8 \%$ & 1.126 & $117.0 \%$ & $\mathrm{t} 3.15$ \\
\hline Matrix 7 & 1486 & $118.2 \%$ & 1936 & $147.5 \%$ & 0.832 & $86.4 \%$ & $\mathrm{t} 3.16$ \\
\hline Mean & 936 & $70.2 \%$ & 1007 & $72.8 \%$ & 0.992 & $103.6 \%$ & $\mathrm{t} 3.17$ \\
\hline $\mathrm{CV}$ & $47.2 \%$ & $51.9 \%$ & $56.6 \%$ & $63.4 \%$ & $9.4 \%$ & $10.2 \%$ & $\mathrm{t} 3.18$ \\
\hline \multirow[t]{3}{*}{ B } & \multicolumn{6}{|c|}{$20 \mathrm{mg} / \mathrm{L}$ vancomycin experiment } & $\begin{array}{l}\mathrm{t} 3.19 \\
\mathrm{t} 3.20\end{array}$ \\
\hline & \multicolumn{2}{|c|}{ Vancomycin } & \multicolumn{2}{|l|}{ IS } & \multicolumn{2}{|l|}{$\mathrm{RR}$} & $\mathrm{t} 3.21$ \\
\hline & Area & $\mathrm{ME}$ & Area & $\mathrm{ME}$ & $\overline{R R}$ & $\mathrm{ME}$ & $\mathrm{t} 3.22$ \\
\hline Matrix 1 & 6735 & & 1768 & - & 3.830 & - & $\mathrm{t} 3.23$ \\
\hline Matrix 2 & 4485 & $66.6 \%$ & 1046 & $59.2 \%$ & 4.311 & $112.6 \%$ & $\mathrm{t} 3.24$ \\
\hline Matrix 3 & 4623 & $68.6 \%$ & 1052 & $59.5 \%$ & 4.360 & $113.9 \%$ & $\mathrm{t} 3.25$ \\
\hline Matrix 4 & 2793 & $41.5 \%$ & 728 & $41.2 \%$ & 3.800 & $99.2 \%$ & $\mathrm{t} 3.26$ \\
\hline Matrix 5 & 5487 & $81.5 \%$ & 1574 & $89.0 \%$ & 3.487 & $91.1 \%$ & $\mathrm{t} 3.27$ \\
\hline Matrix 6 & 2305 & $34.2 \%$ & 571 & $32.3 \%$ & 4.034 & $105.3 \%$ & $\mathrm{t} 3.28$ \\
\hline Matrix 7 & 5782 & $85.9 \%$ & 1803 & $102.0 \%$ & 3.200 & $83.6 \%$ & $\mathrm{t} 3.29$ \\
\hline Mean & 4601 & $63.0 \%$ & 1220 & $63.9 \%$ & 3.860 & $100.9 \%$ & $\mathrm{t} 3.30$ \\
\hline $\mathrm{CV}$ & $34.7 \%$ & $33.3 \%$ & $40.8 \%$ & $42.2 \%$ & $10.9 \%$ & $11.9 \%$ & $\mathrm{t} 3.31$ \\
\hline
\end{tabular}

allow easy and rapid analysis, a substantial between-method difference 365 can be found. Next to standardization differences, plasma components 366 (plasma proteins and salts) can vary between patients and therefore, in- 367 terferences in binding of analytes to the antibody in immunoassays can 368 differ, especially when the analyte is present in low concentrations [29, 369 30]. In our experiment, 3 out of 4 immunoassays showed an acceptable 370 difference with LC-MS/MS (<10\%). Only the Cobas 8000 assay showed 371 a significant proportional difference of $>20.0 \%$.

372

As Cobas 8000 measured invariably higher in the low, therapeutic as 373 well as in the toxic range, there appears to be a standardization differ- 374 ence with the other assays. This difference causes a discordant clinical 375 interpretation in more than 1 out of 5 samples. Given our results, and 376 considering the clear-cut vancomycin therapeutic target concentration 377 ranges, it is likely that clinical dosing decisions may depend on the 378 assay that is used in an individual institution as suggested by Zhao 379 et al. [8]. As it is difficult to trace the analytical details of the methods 380 used for the determination of the therapeutic interval values [10], it 381 remains unclear which of the tested assays gives values most similar 382 to the originally used techniques. Some other studies explored 383 between-assay differences. In this regard, a study by Azzazy et al. com- 384 pared a FPIA method with a HPLC method for vancomycin measure- 385 ment, and found satisfactory agreement between both methods [31]. 386 Bijleveld et al. recently found only small differences in vancomycin 387

Stability of plasma samples. Values are expressed as a \% recovery from the initial value.

\begin{tabular}{|c|c|c|c|c|c|c|c|c|c|}
\hline \multirow[t]{2}{*}{ Storage time } & \multicolumn{3}{|c|}{$\begin{array}{l}\text { Concentration } 1 \\
(8.2 \mathrm{mg} / \mathrm{L})\end{array}$} & \multicolumn{3}{|c|}{$\begin{array}{l}\text { Concentration } 2 \\
(18.2 \mathrm{mg} / \mathrm{L})\end{array}$} & \multicolumn{3}{|c|}{$\begin{array}{l}\text { Concentration } 3 \\
(35.5 \mathrm{mg} / \mathrm{L})\end{array}$} \\
\hline & $+20^{\circ} \mathrm{C}$ & $+8^{\circ} \mathrm{C}$ & $-20^{\circ} \mathrm{C}$ & $+20^{\circ} \mathrm{C}$ & $+8{ }^{\circ} \mathrm{C}$ & $-20^{\circ} \mathrm{C}$ & $+20^{\circ} \mathrm{C}$ & $+8{ }^{\circ} \mathrm{C}$ & $-20^{\circ} \mathrm{C}$ \\
\hline 1 day & 92.9 & 99.5 & 99.0 & 94.5 & 99.5 & 99.6 & 96.7 & 101.5 & 100.7 \\
\hline 2 days & 93.3 & 98.7 & 98.9 & 96.0 & 98.6 & 96.9 & 92.8 & 100.5 & 98.5 \\
\hline 1 week & 93.6 & 96.8 & 97.7 & 98.3 & 100.1 & 99.8 & 97.4 & 97.3 & 97.0 \\
\hline 3 weeks & 96.9 & 92.6 & 94.0 & 93.9 & 93.2 & 95.2 & 90.3 & 99.8 & 99.3 \\
\hline
\end{tabular}


A1

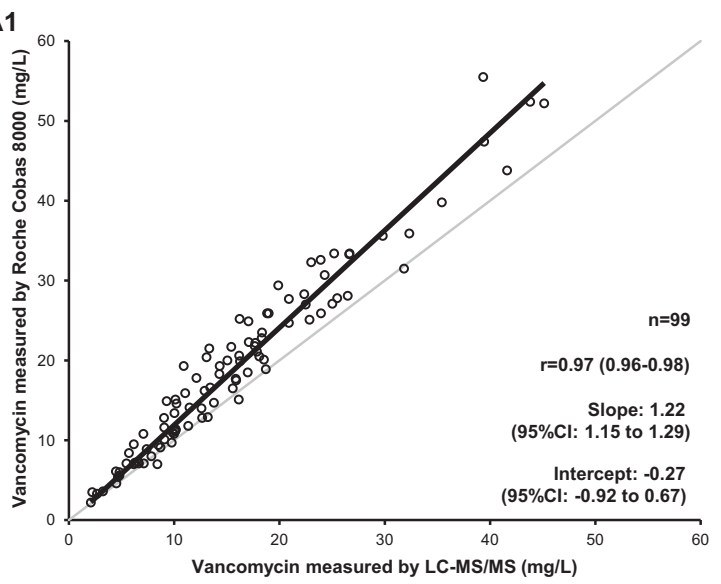

B1

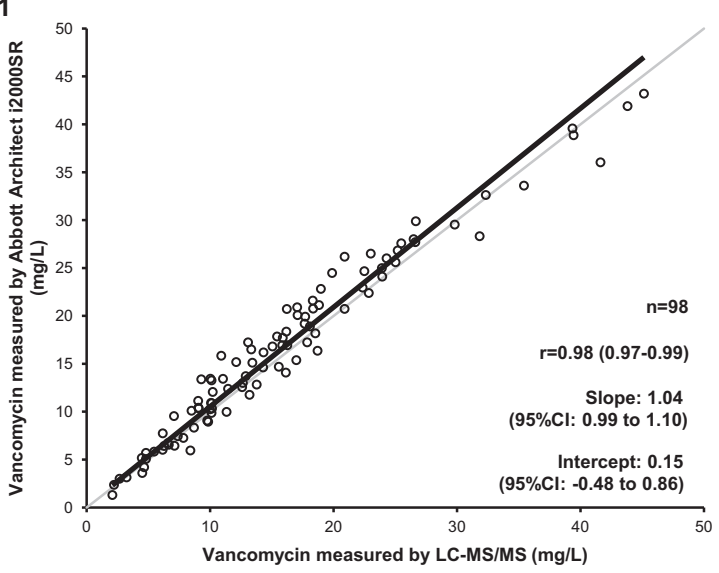

C1
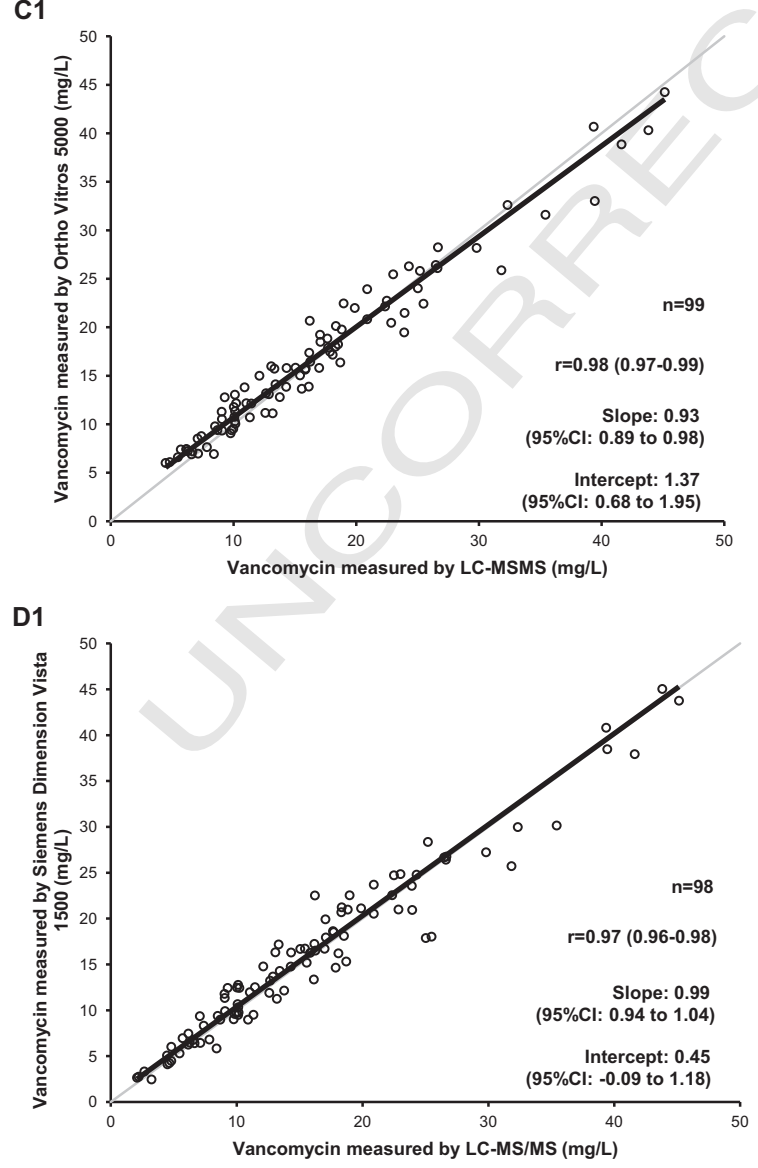
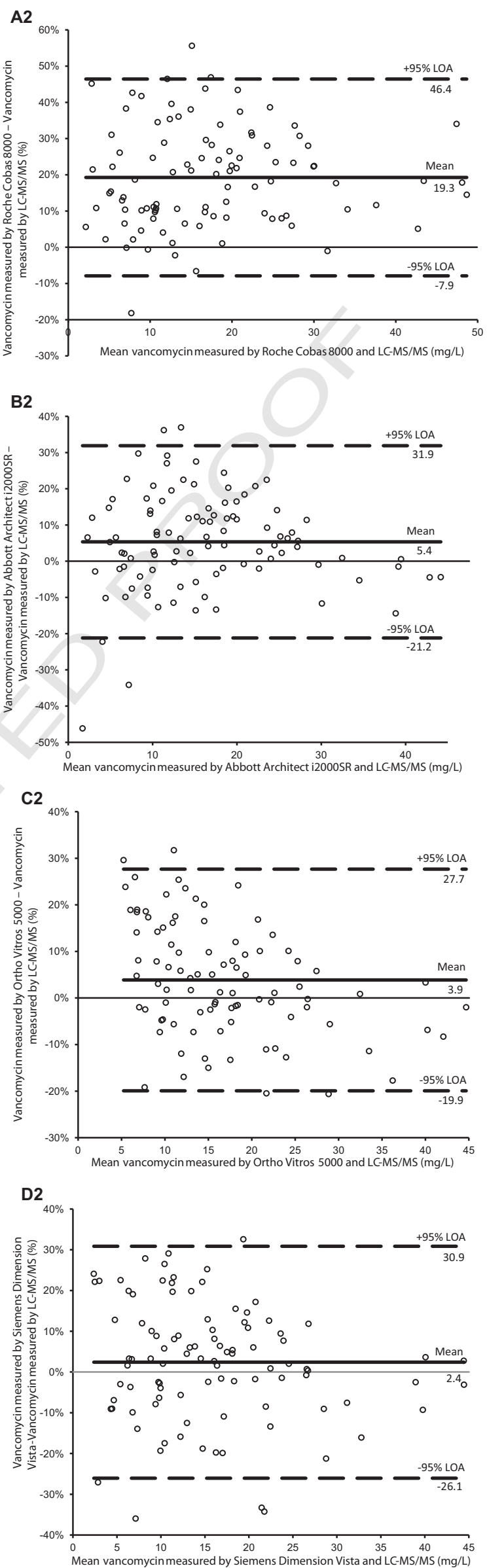


\begin{tabular}{|c|c|c|c|c|}
\hline \multirow{2}{*}{ Discordance $22.2 \%$} & \multicolumn{3}{|c|}{ Roche Cobas 8000} \\
\cline { 3 - 6 } & $<15 \mathrm{mg} / \mathrm{L}$ & $15-25 \mathrm{mg} / \mathrm{L}$ & $>25 \mathrm{mg} / \mathrm{L}$ \\
\hline \multirow{3}{*}{ LC-MS/MS } & $<15 \mathrm{mg} / \mathrm{L}$ & 43 & 10 & 0 \\
\cline { 2 - 5 } & $15-25 \mathrm{mg} / \mathrm{L}$ & 0 & 20 & 12 \\
\cline { 2 - 5 } & $>25 \mathrm{mg} / \mathrm{L}$ & 0 & 0 & 14 \\
\hline
\end{tabular}

B

\begin{tabular}{|c|c|c|c|c|}
\hline \multicolumn{2}{|c|}{ Discordance $12.2 \%$} & \multicolumn{3}{|c|}{ Abbott Architect i2000SR } \\
\cline { 3 - 6 } & $<15 \mathrm{mg} / \mathrm{L}$ & $15-25 \mathrm{mg} / \mathrm{L}$ & $>25 \mathrm{mg} / \mathrm{L}$ \\
\hline \multirow{3}{*}{ LC-MS/MS } & $<15 \mathrm{mg} / \mathrm{L}$ & 45 & 7 & 0 \\
\cline { 2 - 5 } & $15-25 \mathrm{mg} / \mathrm{L}$ & 2 & 27 & 3 \\
\cline { 2 - 5 } & $>25 \mathrm{mg} / \mathrm{L}$ & 0 & 0 & 14 \\
\hline
\end{tabular}

C

\begin{tabular}{|c|c|c|c|c|}
\hline \multicolumn{2}{|c|}{ Discordance $8.1 \%$} & \multicolumn{4}{|c|}{ Ortho Vitros 5000} \\
\cline { 3 - 6 } & $<15 \mathrm{mg} / \mathrm{L}$ & $15-25 \mathrm{mg} / \mathrm{L}$ & $>25 \mathrm{mg} / \mathrm{L}$ \\
\hline \multirow{3}{*}{ LC-MS/MS } & $<15 \mathrm{mg} / \mathrm{L}$ & 50 & 3 & 0 \\
\cline { 2 - 5 } & $15-25 \mathrm{mg} / \mathrm{L}$ & 2 & 28 & 2 \\
\cline { 2 - 5 } & $>25 \mathrm{mg} / \mathrm{L}$ & 0 & 1 & 13 \\
\hline
\end{tabular}

\begin{tabular}{|c|c|c|c|c|}
\hline \multirow{2}{*}{ Discordance 6.1\% } & \multicolumn{3}{|c|}{ Siemens Dimension Vista 1500} \\
\cline { 3 - 6 } & $<15 \mathrm{mg} / \mathrm{L}$ & $15-25 \mathrm{mg} / \mathrm{L}$ & $>25 \mathrm{mg} / \mathrm{L}$ \\
\hline \multirow{3}{*}{ LC-MS/MS } & $<15 \mathrm{mg} / \mathrm{L}$ & 50 & 3 & 0 \\
\cline { 2 - 5 } & $15-25 \mathrm{mg} / \mathrm{L}$ & 2 & 30 & 2 \\
\cline { 2 - 5 } & $>25 \mathrm{mg} / \mathrm{L}$ & 0 & 1 & 13 \\
\hline
\end{tabular}

Fig. 3. Clinical impact of interpretation of the obtained vancomycin concentrations ( $\mathrm{mg} / \mathrm{L}$ ) with A) Roche Cobas 8000; B) Abbott Architect i2000SR; C) Ortho Vitros 5000 and D) Siemens Dimension Vista 1500 compared to LC-MS/MS. Results are presented as cross tables with the number of samples in each clinical interpretative category.

concentrations between their LC-MS/MS method and FPIA assay, with a slightly negative difference ( $-0.9 \%$ (95\%CI: -6.8 to $5.1 \%)$ ) [18]. More recently, Shipkova et al. compared 8 different immunoassays from three different manufacturers for TDM of vancomycin [6]. In their study, they found that all assays showed a proportional difference compared to the results obtained with the Cobas 8000 (12.0-19.0\% lower). This is in accord with our results. Our study, however, adds one manufacturer and is able to give a comparison with reference technology.

We further examined the observed difference by performing a crossanalysis of standards. We analyzed the TDM Preciset (Roche standards) with our LC-MS/MS assay and our LC-MS/MS standards (spiked blank plasma) with the Cobas assay. This analysis, however, didn't produce conclusive evidence. The TDM Preciset standards gave results within $\pm 15 \%$ of the indicated values and the LC-MS/MS standards gave results within $\pm 16 \%$ of the theoretical values. It appears that other factors are the main cause of the observed difference. Roche standards analyzed with Roche reagent on Cobas 8000 c702 might suffer from a differential $\mathrm{ME}$ as compared to true vancomycin patient samples. Also, several structurally related impurities can be present in vancomycin preparations. Diana et al. found 15 different impurities, together composing $16.6 \%$ of the sample [7]. The variation of these impurities with different lots of vancomycin or the impact on different immunoassays has never been examined into detail. As most of these metabolites have substan- 410 tially different molecular masses or show different polarities, LC-MS/ 411 MS methods are least likely to suffer from interference by these 412 compounds. The Roche method might suffer more from interference 413 (or cross-reactivity) by these components or other substances present 414 in samples from patients receiving vancomycin. A limitation of our 415 study, however, is that lot-to-lot variability of the different assays was 416 not included. Other lots of calibrators and reagents might give rise to 417 slightly different results.

In conclusion, we developed an applicable LC-MS/MS method for 419 vancomycin measurement in human plasma. Correlation of our 420 LC-MS/MS method with four immunoassays showed substantial differ- 421 ences with the Cobas 8000 assay, causing discordant clinical interpreta- 422 tion in more than 1 out of 5 samples. Therefore the transferability of 423 vancomycin results between laboratories has to be interpreted with 424 caution.

Supplementary data to this article can be found online at http://dx. 426 doi.org/10.1016/j.cca.2014.12.012.

\section{Uncited reference}

\section{Acknowledgments}

We kindly thank Pharm. Clin. Biol. E. Jacobs of the H. Hart Hospital 431 Lier for assistance with Ortho Vitros 5000. Karel Allegaert, Pieter 432 Vermeersch (fundamental clinical investigatorship $1800214 \mathrm{~N}$ and 433 $1842013 \mathrm{~N}$, respectively) and Steven Pauwels (Clinical fellowship 434 $1700314 N$ ) are supported by the Fund for Scientific Research, Flanders. 435 Isabel Spriet has clinical research funding from the University Hospitals 436 Leuven.

\section{References}

[1] Finch RG, Eliopoulos GM. Safety and efficacy of glycopeptide antibiotics. J Antimicrob 439 Chemother 2005;55(Suppl. 2):ii5-ii13.

[2] Steinkraus G, White R, Friedrich L. Vancomycin MIC creep in non-vancomycin- 441 intermediate Staphylococcus aureus (VISA), vancomycin-susceptible clinical 442 methicillin-resistant $S$, aureus (MRSA blood isolates from 2001-05). J Antimicrob 443 Chemother 2007;60:788-94.

[3] Gould IM. Clinical relevance of increasing glycopeptide MICs against Staphylococcus 445 aureus. Int J Antimicrob Agents 2008;31(Suppl. 2):1-9.

[4] Wilson JF, Davis AC, Tobin CM. Evaluation of commercial assays for vancomycin and 447 aminoglycosides in serum: a comparison of accuracy and precision based on exter- 448 nal quality assessment. J Antimicrob Chemother 2003;52:78-82. 449

[5] König K, Kobold U, Fink G, et al. Quantification of vancomycin in human serum by 450 LC-MS/MS. Clin Chem Lab Med 2014;51:1761-9.

[6] Shipkova M, Petrova D, Rosler A, et al. Comparability and imprecision of 8 frequently 452 used commercially available immunoassays for therapeutic drug monitoring. Ther 453 Drug Monit 2014;36:433-41.

[7] Diana J, Visky D, Hoogmartens J, Van Schepdael A, Adams E. Investigation of 455 vancomycin and related substances by liquid chromatography/ion trap mass 456 spectrometry. Rapid Commun Mass Spectrom 2006;20:685-93.

[8] Zhao W, Kaguelidou F, Biran V, et al. External evaluation of population pharmacoki- 458 netic models of vancomycin in neonates: the transferability of published models to 459 different clinical settings. Br J Pharmacol 2012;75:1068-80.

[9] Zhao W, Jacqz-Aigrain E. The importance of knowing how vancomycin is measured 461 when interpreting its pharmacokinetic results. Ther Drug Monit 2013;35:416. 462

[10] Kullar R, Davis SL, Levine DP, Rybak MJ. Impact of vancomycin exposure on out- 463 comes in patients with methicillin-resistant Staphylococcus aureus bacteremia: sup- 464 port for consensus guidelines suggest targets. Clin Infect Dis 2011;52:975-81. 465

[11] Rybak M, Momaestro B, Rotschafer JC, et al. Therapeutic drug monitoring of vanco- 466 mycin in adult patients: a consensus review of the American Society of Health- 467 System Pharmacists, the Infectious Diseases Society of America, and the Society of 468 Infectious Diseases Pharmacists. Am J Health Syst Pharm 2009;66:82-98. 469

[12] Mouton JW, Theuretzbacher U, Craig WA, Tulkens PM, Derendorf H, Cars O. Tissue 470 concentrations: do we ever learn? J Antimicrob Chemother 2008;61:235-7. 471

[13] Lamer C, de Beco V, Soler P, et al. Analysis of vancomycin entry into pulmonary lin- 472 ing fluid by bronchoalveolar lavage in critically ill patients. Antimicrob Agents 473 Chemother 1993;37:281-6. 
[14] Rybak MJ. The pharmacokinetic and pharmacodynamic properties of vancomycin. Clin Infect Dis 2006;42(Suppl.1):S35-9.

[15] Cass RT, Villa JS, Karr DE, Schmidt Jr DE. Rapid bioanalysis of vancomycin in serum and urine by high-performance liquid chromatography tandem mass spectrometry using on-line sample extraction and parallel analytical columns. Rapid Commun Mass Spectrom 2001;15:406-12.

[16] Zhang T, Watson DG, Azike C, et al. Determination of vancomycin in serum by liquid chromatography-high resolution full scan mass spectrometry. J Chromatogr B 2007; 857:352-6.

[17] Shibata N, Ishida M, Prasad YV, Gao W, Yoshikawa Y, Takada K. Highly sensitive quantification of vancomycin in plasma samples using liquid chromatography-tandem mass spectrometry and oral bioavailability in rats. J Chromatogr B 2003;787: 211-8.

[18] Bijleveld Y, de Haan T, Toersche J, et al. A simple quantitative method analyzing amikacin, gentamicin, and vancomycin levels in human newborn plasma using ion-pair liquid chromatography/tandem mass spectrometry and its applicability to a clinical study. J Chromatogr B 2014;951:110-8.

[19] CLSI. Evaluation of precision performance of quantitative measurement methods; approved guideline-second edition. CLSI Document EP05-A2. Wayne, PA: Clinical Laboratory Standards Institute; 2004.

[20] European Medicines Agency, guideline on bioanalytical method validation. 21 July $2011 ; 2011$.
[21] CLSI. Mass spectrometry in the clinical laboratory: general principles and guidance; 497 approved guideline. CLSI Document C50-A. Wayne, PA: Clinical Laboratory Stan- 498 dards Institute; 2007

[22] Kees MG, Wicha SG, Seefeld A, Kees F, Kloft C. Unbound fraction of vancomycin in 500 intensive care unit patients. J Clin Pharmacol 2014;54:318-23. 501

[23] Abbott Architect iVancomycin G3, package insert; 11-2012. 502

[24] Roche Vancomycin VANC2, package insert 08-2013,V2; 2013.

[25] Ortho Vitros 5000 Vancomycin VANC, package insert 05-2012, V4; 2012.

[26] Siemens Dimension Vista Vancomycin VANC, package insert; 11-2012. 505

[27] Bland JM, Altman DG. Statistical methods for assessing agreement between two 506 methods of clinical measurement. Lancet 1986;1:307-10. 507

[28] Passing H, Bablok W. A new biometrical procedure for testing equality of measure- 508 ments from two different analytical methods. J Clin Chem Clin Biochem 1983;21: 509 709-20.

29] Ezan A, Emmanuel A, Valente D, Grognet JM. Effect of variability of plasma interfer- 511 ences on the accuracy of drug immunoassays. Ther Drug Monit 1997;19:212-8. 512

[30] LeGatt DF, Blakney GB, Higgins TN, et al. The effect of paraproteins and rheumatoid 513 factor on four commercial immunoassays for vancomycin: implications for 514 laboratorians and other health care professionals. Ther Drug Monit 2012;34:306-11. 515

[31] Azzazy HM, Chou PP, Tsushima JH, et al. Abbott AxSYM vancomycin II assay: multi- 516 center evaluation and interference studies. Ther Drug Monit 1998;20:202-8. 